Results The overall estimated HIV prevalence was $6.7 \%$ (95\% confidence interval [CI]: $6.2 \%-7.2 \%)$ and the syphilis prevalence was $3.9 \%$ (95\% CI: $3.5 \%-4.2 \%$ ). In 2004 , about $10.3 \%$ of the MSM were 18 and 19 year old; this proportion had significantly increased to $17.6 \%(p<0.01)$ in 2011. Similarly, the proportion of MSM aged 35 years and above increased significantly from 2004 to 2011 (7.5\% vs $12.8 \%, \mathrm{p}<0.01)$. The estimated HIV prevalence in 2004 was $10.6 \%$ (95\% CI: $8.4 \%-12.7 \%$ ), this reduced to $2.8 \%$ (95\% CI: $2.1 \%-3.5 \%$, $\mathrm{p}<0.01)$. The prevalence of syphilis in 2004 was $11.4 \%$ (95\% CI: $9.1 \%-13.6 \%$ ), this reduced to $0.4 \%$ (95\% CI: $0.1 \%-0.7 \%, p=35$ years, OR: 9.9, 95\% CI: $6.7-14.6$ ) and syphilis reactivity (OR: 3.1, 95\% CI: $2.4-3.9$ ).

Conclusions The HIV and syphilis prevalence declined over the eight year period. A higher proportion of younger (18/19 year old), and MSM older than 35 are accessing HIV testing services; thus we need to develop intervention programmes for them.

\section{P3.120 RISK FACTORS, ANTIMICROBIAL SUSCEPTIBILITY PROFILES AND PUBLIC HEALTH IMPLICATIONS OF PATIENTS PRESENTING WITH PHARYNGEAL GONOCOCCAL INFECTION IN ENGLAND AND WALES}

doi:10.1136/sextrans-2013-051184.0579

\begin{abstract}
C M Obi, G Hughes, C Ison, S Chisholm. Health Protection Agency, London, UK
\end{abstract}
Background Pharyngeal infection with Neisseria gonorrhoeae is usually asymptomatic and often under-diagnosed. It could therefore be an important source of ongoing transmission particularly among men who have sex with men (MSM). We investigated the prevalence and risk factors of pharyngeal infection among MSM diagnosed with gonorrhoea, and associated antimicrobial susceptibility profiles to cefixime and ceftriaxone.

Method Data from patients included in the national Gonococcal Resistance to Antimicrobials Surveillance Programme (GRASP) from 2007-2010 and self-identified as MSM were analysed. Risk factors for pharyngeal-only infection (POI) versus rectal and/or genital, infections, or a combination (RGI), were examined using multivariable logistic regression adjusting for age, clinic and year of attendance. Minimum inhibitory concentrations (MICs) of cefixime and ceftriaxone at different sites were compared using logistic regression. Results Of the 2249 MSM in this analysis, 202 (8\%) were diagnosed with a POI. They were significantly more likely to have multiple sexual partners $(\mathrm{OR}=1.44$, CI $1.0-2.1)$ and asymptomatic infection $(\mathrm{OR}=13.4$, CI 9.0-20.0); but less likely to report a history of gonorrhoea $(\mathrm{OR}=0.61 \mathrm{CI} 0.4-0.8)$; have a concurrent STI $(\mathrm{OR}=0.50$, CI 0.3-0.8) or be HIV-positive $(\mathrm{OR}=0.47$, CI 0.3-0.9), when compared to those with RGI. 168 (82\%) of POI and 2047 ( $89 \%$ ) of RGI cases had isolates retrieved for antimicrobial sensitivity testing. There was a two-fold increase in the geometric mean MIC of cefixime and ceftriaxone required to inhibit growth of $N$. gonorrheae resident in the pharynx compared to other sites $(\mathrm{OR}=2.14$; CI 1.2-3.8; $\mathrm{OR}=2.21$; CI 1.2-4.0; $\mathrm{p}<0.05)$ although the proportion of isolates exhibiting decreased susceptibility to cefixime and ceftriaxone at MIC $\geq 0.125 \mathrm{mg} / 1$ did not vary significantly.

Conclusions Patients with POI have high rates of partner change and may be at increased risk of transmitting less sensitive gonococcal strains. Screening for pharyngeal infection should be intensified. Tests of cure should be performed routinely to ensure successful treatment.

\section{P3.121 ARE WE SEEING A TRUE RISE IN NEISSERIA GONORRHOEAE AND CHLAMYDIA TRACHOMATIS IN ENGLAND IN MEN WHO HAVE SEX WITH MEN?}

doi:10.1136/sextrans-2013-051184.0580

G Haidari, M E 0 Perry, J A White. Guys and St Thomas' NHS Foundation Trust, London, UK
Background The Health Protection Agency (HPA) in England have reported the number of sexually transmitted infections (STIs) diagnosed in men who have sex with men (MSM) continues to rise. Of note, Genitourinary medicine (GUM) clinic data for 2011 showed gonorrhoea (GC) diagnoses in MSM had increased by $61 \%$ and chlamydia (CT) diagnoses by $48 \%$ compared to 2010 figures. A number of factors may have contributed, including increased MSM screening and wider use of highly sensitive nucleic acid amplification tests (NAATs), especially when testing rectal \& pharyngeal sites in MSM. In our London clinic, extragenital testing with dual GC/CT NAATs has been routine since 2009 in MSM.

Aim We hypothesise there is no true rise in incidence in MSM and that recent changes are a reflection of increased testing and widespread use of NAATs.

Methods Retrospective annual comparison of all positive MSM GC \& CT infections identified by Aptima Combo2 (AC2; GenProbe) between $2010 \& 2012$. The number of GC cultures and positivity rates were also compared.

Results

Abstract P3.121 Table 1 Comparison of total MSM GC and CT infections seen in 2010, 2011 and 2012.

\begin{tabular}{|c|c|c|c|c|c|c|}
\hline & \multicolumn{2}{|c|}{$\begin{array}{l}2010 \\
(n=5570)\end{array}$} & \multicolumn{2}{|c|}{$\begin{array}{l}2011 \\
(n=6292)\end{array}$} & \multicolumn{2}{|c|}{$\begin{array}{l}2012 \\
(n=6843)\end{array}$} \\
\hline & CT & GC & CT & GC & CT & GC \\
\hline $\begin{array}{l}\text { Total number of CT and GC cases } \\
\text { (infected at urethra, rectum and/ } \\
\text { or throat) }\end{array}$ & 570 & 644 & 711 & 692 & 726 & 881 \\
\hline $\begin{array}{l}\text { Positivity rate }(\% \text { of MSM tested } \\
\text { who were + ve for } \mathrm{GC} / \mathrm{CT})\end{array}$ & $12 \%$ & $10 \%$ & $11 \%$ & $11 \%$ & $11 \%$ & $11 \%$ \\
\hline
\end{tabular}

When comparing the positivity rates of GC \& CT in MSM seen for testing at our clinics there has not been a significant rise from 2010 to 2012. Actual numbers of MSM with infections are higher; however this seems to reflect an increase in testing episodes only.

Conclusion According to GUMCAD returns our clinic cohort accounts for $13 \%$ of GC and 8\% CT cases seen in English MSM; thus we would expect any dramatic increase in incidence to be reflected in our data, at least for the London area. Using our somewhat crude analysis we suggest the true increases in GC and CT infections reported among MSM in 2011 are significantly more modest than the rise suggested by HPA data, and most likely reflects the uptake nationally of 3-site NAAT screening.

\section{P3.122* HEPATITIS B INCIDENCE 2002-2012 AND FALLING LEVELS OF IMMUNITY IN MEN WHO HAVE SEX WITH MEN (MSM) AT SEXUAL HEALTH SERVICE, MELBOURNE}

doi:10.1136/sextrans-2013-051184.0581

${ }^{1,2,3} \mathrm{~N}$ S Gamagedara, ${ }^{1,2,3} \mathrm{~A}$ P Weerakoon, ${ }^{1,3} \mathbf{H}$ Zou, ${ }^{1,3} \mathrm{G}$ Fehler, ${ }^{1,3} \mathrm{M}$ Y Chen, ${ }^{1,3}$ T R H Read, ${ }^{1,3} \mathrm{C}$ S Bradshaw, ${ }^{1,3} \mathrm{C}$ K Fairley. ${ }^{1}$ Melbourne Sexual Health Centre, Alfred Hospital, Melbourne, Australia, 'Department Health Services, Ministry of Health, Colombo, Sri Lanka, ${ }^{3}$ School of Population Health, University of Melbourne, Melbourne, Australia

Background Incidence of hepatitis B has not been well studied in men who have sex with men (MSM) recently despite increases in sexual risk practises and sexually transmitted infections (STI). We determined the level of immunity to hepatitis $B$ and the incidence of hepatitis B infection among MSM attending a sexual health service during last 10 years.

Methods The study involved a cross sectional analysis of all MSM attending Melbourne Sexual Health Centre (MSHC)during 1st July 2002 - 30th June 2012 and a retrospective cohort study of MSM who had attended MSHC on multiple occasions and had hepatitis serology done more than once. 